NASZA DERMATOLOGIA Online OUR DERMATOLOGY Online Nil

Competing Interests: None

\section{LICHENOID REACTIONS IN RED TATTOO: REPORT OF 2 CASES}

\section{César Bimbi}

Brazilian Society of Dermatology, Andradas 1464 / 112, Porto Alegre, Brazil

Corresponding author: Dr César Bimbi

cbimbi@terra.com.br

\begin{abstract}
Complications of cosmetic decorative tattoos were uncommon some decades ago. The practice is increasing and more cases are being reported. Red pigments are by far the commonest cause in tattoo reactions. We report two cases of lichenoid reactions limited to red tattoo pigment and review the literature on the subject.
\end{abstract}

Key words: red tattoo; lichenoid reaction; tattoo reactions

\section{Introduction}

Artistic tattoos rely on the use of numerous pigments (including red) which are not all entirely inert once placed in the dermis. Allergic reactions to a particular pigment can manifest in several ways including lichenoid reaction, allergic contact dermatitis and photoallergic dermatitis, granulomatous or pseudolymphoma reactions.

We have described two cases of lichenoid reaction developing to red ink in colored tattoos.

\section{Case Report}

\section{Case 1}

A healthy 36 -year-old man presented with a 5 month history of plaque lesions inside the tatoo areas of the body (Fig. 1, 2) corresponding specifically to the areas with red pigment. The remainder of the tattoo was unaffected. On examination of the left deltoid area and right leg, indurated plaques involving red coloured areas of the tattoo were shown. These lesions were firm and the edges were warty, bearing clinical similarity to hypertrophic lichen planus.

Incisional biopsy revealed histological features of lichen planus with dense lynphohistiocytic infiltrate in the papillary dermis and no evidence of granulomatous inflammation.

\section{Case 2}

A 25-year-old female presented with a complaint of pruritic raised lesion in a tattoo drawn over her upper dorso over the past 3 weeks (Fig. 3a, b). The lesion appeared over the site of a tattoo that was injected one month back. She had no history of cough or weight loss. Biopsy was not taken, corticosteroid cream was indicated first.

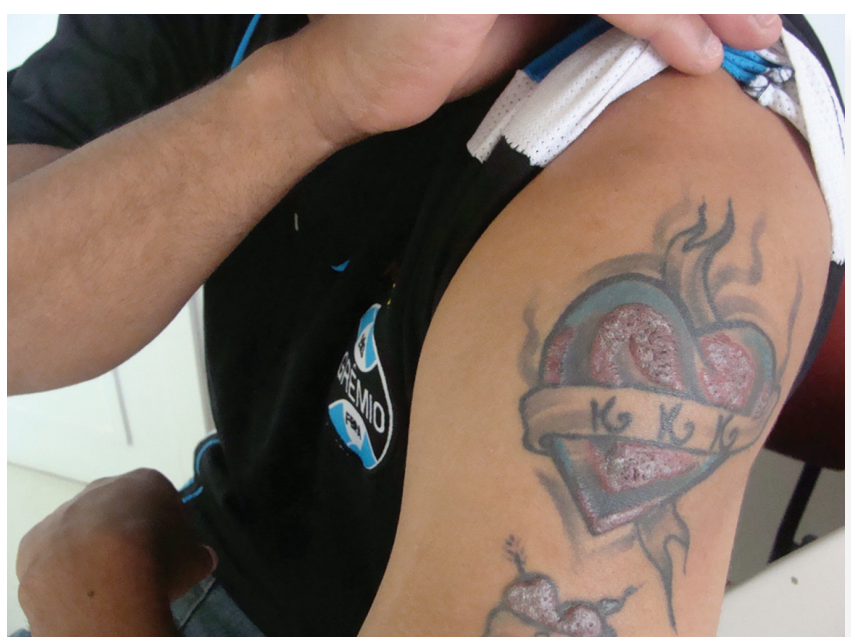

Figure 1. Verrrucous plaques over the red ink with sparing of the blue component.

\section{Discussion}

Complications of decorative tatoos were considered rare some decades ago [5]. A British report about two cases presented to Clinical Meeting at St John's Hospital Dermatological Society in December 1977 [6], informed that, at that time, a review in the literature made by the authors discovered only four other patients who had lichen restricted to red tattoo áreas. Lichen planus in tattoos was first mentioned by Rook and Thomas in 1952 [1]. With the popularity of tatooing practices worldwide, more adverse skin reactions are being reported. $24 \%$ of the US population has tattoos [2]. 


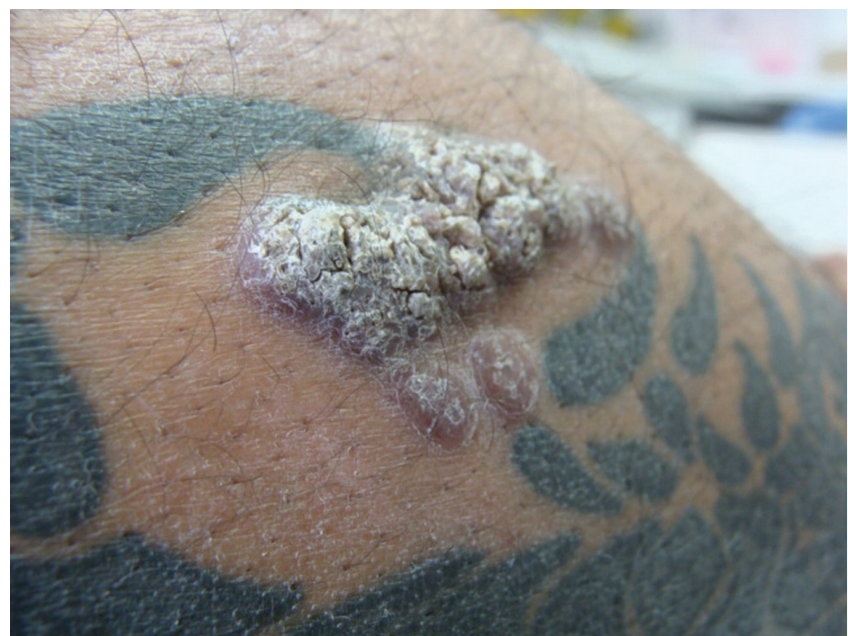

Figure 2. Right leg, same patient. Indurated verrucous nodules over the red component of the tattoo (scorpion tail), blue ink also spared.

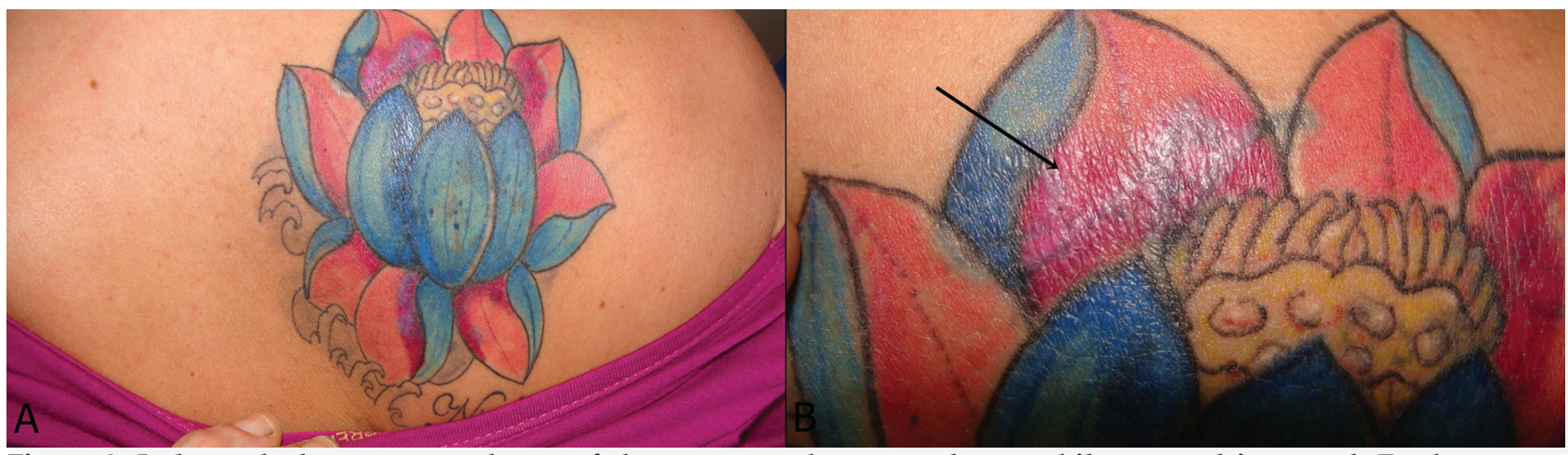

Figure 3. Indurated plaque over red part of the tattoo on the upper dorso, while non-red is spared. Erythematous nodules on petals of the flower.

Reactions in red ink tattoos are increasingly being recorded in current literature.

Red in tattoos has been far more complicated than other colours. Observing the photos among all reports, there is a striking similarity [5]; all show features of elevated nodules or plaques confined to red. Mercury contained in red ink (mercuric sulphide or cinnabar) has been well recognized in the past as the causative agent in reactions associated with red tattoos. Chemical substitutes for mercury are the modern alternatives: sienna-ferric hydrate, cadmium-selenide and organic vegetable dyes, sandalwood and brazilwood, and have largely replaced the use of mercury, but sensitivity to red dyes still occur. Most tattooists do not know the composition of the pigments that they use. It is not easy to define the chemicals involved. In fact, the compositions of many inks have been identified. However, as new mixtures are created, it becomes difficult to identify the specific ingredients in a particular ink. There are also many reports in the literature of reactions to purple, light blue, green, black and yellow pigments. The exact incidence is unknown. The risk of dangerous chemicals in tattoos seems to be underestimated. Engel et all. [3] adverts the alarming fact that in Europe, the same azo pigments that are forbidden to be used in cosmetics only applied to the skin surface, are "allowed" (no regulations) in tattooing. So, heavy amounts of up to hundreds of milligrams of carcinogenic aromatic amines are directly injected into the skin. Currently data is lacking regarding the safety of tattoo pigment ingredients. Also, none of the tattoo ink or additives are FDA approved [4].

\section{REFERENCES}

1. Rook AJ, Thomas PJB. Social and medical aspects of tattooing. Practitioner. 1952;169:60

2. Laumann AE, Derick AJ. Tattoos and body piercings in the United States: A national data set. J Am Acad Dermatol. 2006;55:413-21.

3. Engel E, Santarelli F, Vasold R, Maisch T, Ulrich H, Prantl L, et al. Modern tattoos cause high concentrations of hazardous pigments in skin. Contact Dermatitis. 2008.58:228-33.

4. Leffingwell H. Cosmetics Legislation. (available from www. leffingwell.com/cosmetics/vol_1en.pdf). Accessed on October 2007. 5. Winkelmann RK, Harris RB. Lichenoid delayed hypersensitivity reactions in tattoos. J Cutan Pathol. 1979;6:59-65.

6. Clarke J, Black MM. Lichenoid tattoo reactions. Br J Dermatol $1979 ; 100: 451-4$

Copyright by César Bimbi. This is an open access article distributed under the terms of the Creative Commons Attribution License, which permits unrestricted use, distribution, and reproduction in any medium, provided the original author and source are credited. 